\title{
Trauma and dissociation in a Chinese-American sample
}

\author{
Colin A. Ross* and Cathy Kuo \\ The Colin A. Ross Institute for Psychological Trauma, 1701 Gateway, \#349, Richardson, TX 75080 USA
}

\begin{abstract}
Although there have been studies of the rates of reported childhood trauma and dissociation in China, no studies have compared the rates in China and among Chinese-Americans using the same measures. The authors sought to compare the frequency of reported childhood trauma and dissociative disorders in a sample of factory workers in Shanghai and a non-clinical Chinese-American sample in Dallas. The authors interviewed 58 Chinese-American adults with the Dissociative Experiences Scale and the Dissociative Disorders Interview Schedule and then compared the results to those for 618 factory workers in Shanghai. The two groups did not differ on dissociative experiences or disorders except that $5.2 \%$ of the US group met criteria for dissociative disorder not otherwise specified compared to none of the Shanghai participants: of the US participants, $5.2 \%$ reported childhood physical and/or sexual abuse compared to $0.2 \%$ of the Shanghai participants. Both groups reported substantially lower rates of childhood physical and sexual abuse than child and adolescent participants in surveys in China and Taiwan. The findings raise two questions: have the rates of childhood trauma increased in China in the last three or four decades; and, do Chinese-American adults report more childhood trauma than adults in China?
\end{abstract}

\section{Introduction}

A series of studies [1-7] has shown that dissociative symptoms and disorders can be detected in clinical populations in China with good reliability. Additionally, a number of studies have demonstrated that childhood maltreatment is not rare in non-clinical populations in China [8-11].

Chen, Dunne and Han [8] found in a survey of 2,300 high school students in four provinces in China that $16.7 \%$ of females and $10.5 \%$ of males reported an unwanted sexual experience prior to age 16. Although sexual penetration was rare (1\%), at least one type of unwanted physical sexual contact was reported by $8.9 \%$ of females and $5.0 \%$ of males. Li et al. [9] found in a study of 485 Chinese adolescents that $18.8 \%$ reported emotional abuse, $11.1 \%$ physical abuse, $27.0 \%$ sexual abuse, $49.5 \%$ emotional neglect, and 68.7\% physical neglect. Lin et al. [10] found in a sample of 683 rural Chinese children and adolescents age eight to 18 that $21.5 \%$ of girls and $14.2 \%$ of boys reported sexual abuse before age 16. Shen, Zhang, Liang, \& Zhang [11] found in a study of 736 Chinese junior school students that $22.8 \%$ reported emotional neglect, $38.0 \%$ physical neglect, $11.0 \%$ sexual abuse, $10.6 \%$ emotional abuse, and $7.1 \%$ physical abuse. Yen et al. [12], in a study of 1,684 adolescents in rural Taiwan, found that $22.2 \%$ reported childhood physical abuse and $2.5 \%$ reported sexual abuse.

In contrast, Ross et al. [2] reported that, in a sample of 618 factory workers in Shanghai with an average age of 41.8 years, none reported childhood sexual abuse and one reported childhood physical abuse. It is possible that there were false negatives in this sample due to reticence to report abuse, but it is also possible that the participants' reporting was accurate. Given the much higher rates of physical and sexual abuse reported by younger people in China in other studies, one must also consider the possibility that rates of child abuse have increased substantially in China over the last thirty years.

In order to investigate the very low rates of childhood physical and sexual abuse in the sample of Shanghai factory workers, we decided to interview a group of Chinese adults living in the United States with the same measures used by Ross et al. [2]. We hypothesized that there would be no significant difference in rates of reported trauma in the US sample compared to the Shanghai sample. We also hypothesized that there would be no difference in rates of dissociative symptoms and disorders between the two samples. If our hypotheses were supported by our data, the results would be consistent with the low rates of reported childhood trauma in the Shanghai factory workers being accurate; this in turn would be consistent with an increase in rates of childhood trauma in China in the last three or four decades.

\section{Methods \\ Participants}

Participants in Dallas, USA were 58 individuals of Chinese ancestry; 32 were attendees at a non-clinical day program for ChineseAmerican retired people; their ages ranged from 61 to over 80 years; all were fluent in spoken and written Chinese and all their interviews were conducted in Chinese. The facility provides activities, companionship, medical support and transportation for the attendees. Participants were approached consecutively and those who agreed to participate were interviewed. The other 26 participants were still working and their ages ranged from 20 to 60; their interviews were conducted in English at a variety of locations; they were a convenience sample of individuals known to the authors or to other participants; 11 of these 26 individuals were born in the United States. Of the 58 participants, $43(74.1 \%)$ were female, 11 were born in the US, 29 in Taiwan, and 18 in China. The average age was 63.1 years $(\mathrm{SD}=22.4), 24(41.4 \%)$ were

Correspondence to: Colin A. Ross, The Colin A. Ross Institute for Psychological Trauma, 1701 Gateway, \#349, Richardson, TX 75080, USA; E-mail: rossinst@rossinst.com

Key words: trauma, dissociation, Chinese and Chinese-American samples

Received: January 13, 2016; Accepted: February 17, 2016; Published: February 22,2016 
married, and the average number of children per participant was 2.2.

Participants in Shanghai were 618 factory workers reported in a previous study (Xiao, et al., 2004). All workers approached for an interview completed the interview. Their average age was 41.8 years $(\mathrm{SD}=5.9), 321(51.9 \%)$ were female, $570(92.2 \%)$ were married, and the average number of children was 2.0. All participants gave written informed consent. The study was approved by the Internal Review Board at Shanghai Mental Health Center. All interviews were conducted in Chinese, and all participants gave written informed consent.

\section{Measures}

The Dissociative Experiences Scale (DES) is a 28-item self-report measure that has been used in a large number of studies [13-15]. It has good reliability and validity and yields an overall score that ranges from zero to 100 .

The Dissociative Disorders Interview Schedule (DDIS) is a structured interview with good reliability and concurrent validity that has been used in a number of different studies [3,5,16-19]. The DDIS makes DSM-IV [20] diagnoses of somatization disorder, major depressive episode, borderline personality disorder and the five dissociative disorders. It also inquires about substance abuse, childhood physical and sexual abuse, Schneiderian first rank symptoms, secondary features of dissociative identity disorder and $\mathrm{ESP} /$ paranormal experiences.

\section{Data analysis}

The Dallas and Shanghai participants were compared on all variables using $t$ tests for continuous variables and Chi square tests for dichotomous variables, with significance set at $\mathrm{p}=0.05$.

\section{Results}

The Dallas and Shanghai participants differed on age $(t=18.005$, $\mathrm{df}=674, \mathrm{p}<0.001)$; gender $(\mathrm{t}=253, \mathrm{df}=674, \mathrm{p}<0.001)$; percentage married $(\mathrm{t}=12.586, \mathrm{df}=674, \mathrm{p}<0.001)$; and number of children $(\mathrm{t}=2.465, \mathrm{df}=674$, $\mathrm{p}=0.02$ ).

The Dallas and Shanghai participants did not differ significantly from each other on most of the symptom variables (Table 1 and 2), but did differ on trauma histories (Table 3 ).

\section{Discussion}

Our findings were not conclusive concerning our two hypotheses. As predicted, the two groups did not differ on DES scores, secondary features of DID on the DDIS, or rates of the four defined DSM-IV

Table 1. Symptom clusters among factory workers in Shanghai and Chinese-Americans in Dallas, USA.

\begin{tabular}{|l|l|l|l|l|l|}
\hline Symptoms & $\begin{array}{l}\text { Dallas } \\
\text { (n=58) } \\
\text { Average (SD) }\end{array}$ & $\begin{array}{l}\text { Shanghai } \\
\text { (n=618) } \\
\text { Average } \\
\text { (SD) }\end{array}$ & t & df & p \\
\hline DES & $3.8(3.6)$ & $2.6(4.3)$ & 1.923 & 674 & 0.06 \\
\hline Somatic & $2.3(3.5)$ & $2.0(2.5)$ & 1.095 & 674 & 0.27 \\
\hline Schneiderian & $0.1(0.3)$ & $0.01(0.2)$ & 2.195 & 674 & 0.03 \\
\hline $2^{0}$ DID & $0.4(0.6)$ & $0.5(0.8)$ & 0.887 & 674 & 0.38 \\
\hline Borderline & $0.2(0.6)$ & $0.2(0.6)$ & 0.217 & 674 & 0.83 \\
\hline ESP/paranormal & $0.4(0.9)$ & $0.2(0.6)$ & 2.408 & 674 & 0.02 \\
\hline
\end{tabular}

- $\mathrm{DES}=$ Dissociative Experiences Scale; $2^{0}$ DID $=$ secondary features of dissociative identity disorder; ESP = extrasensory perception
Table 2. Diagnoses among factory workers in Shanghai and Chinese-Americans in Dallas, USA.

\begin{tabular}{|c|c|c|c|c|}
\hline Diagnosis & $\begin{array}{l}\text { Dallas } \\
(n=58) \\
N \text { for positive }\end{array}$ & $\begin{array}{l}\text { Shanghai } \\
(n=618) \\
\text { N for positive }\end{array}$ & $\mathbf{X}^{2}$ & $\mathbf{p}$ \\
\hline Amnesia & 0 & 1 & 0.759 & 0.91 \\
\hline Fugue & 0 & 0 & - & 1.00 \\
\hline Depersonalization & 0 & 1 & 0.759 & 0.91 \\
\hline DID & 0 & 0 & - & 1.00 \\
\hline DDNOS & 3 & 0 & 32.108 & $<0.001$ \\
\hline Somatization & 0 & 0 & - & 1.00 \\
\hline Major depression & 7 & 16 & 14.999 & $<0.001$ \\
\hline Borderline & 0 & 2 & 0.188 & 0.84 \\
\hline
\end{tabular}

* DID $=$ dissociative identity disorder; DDNOS $=$ dissociative disorder not otherwise specified

Table 3. Childhood trauma histories among factory workers in Shanghai and ChineseAmericans in Dallas, USA.

\begin{tabular}{|l|l|l|l|l|}
\hline Trauma & $\begin{array}{l}\text { Dallas } \\
\text { (n=58) } \\
\text { N for positive }\end{array}$ & $\begin{array}{l}\text { Shanghai } \\
(\mathbf{n = 6 1 8 )} \\
\text { N for positive }\end{array}$ & $\mathbf{X}^{\mathbf{2}}$ & $\mathbf{p}$ \\
\hline Sexual abuse & 1 & 0 & 10.671 & 0.09 \\
\hline Physical abuse & 2 & 1 & 12.962 & 0.02 \\
\hline $\begin{array}{l}\text { Physical and/or } \\
\text { sexual abuse }\end{array}$ & 3 & 1 & 22.632 & 0.003 \\
\hline
\end{tabular}

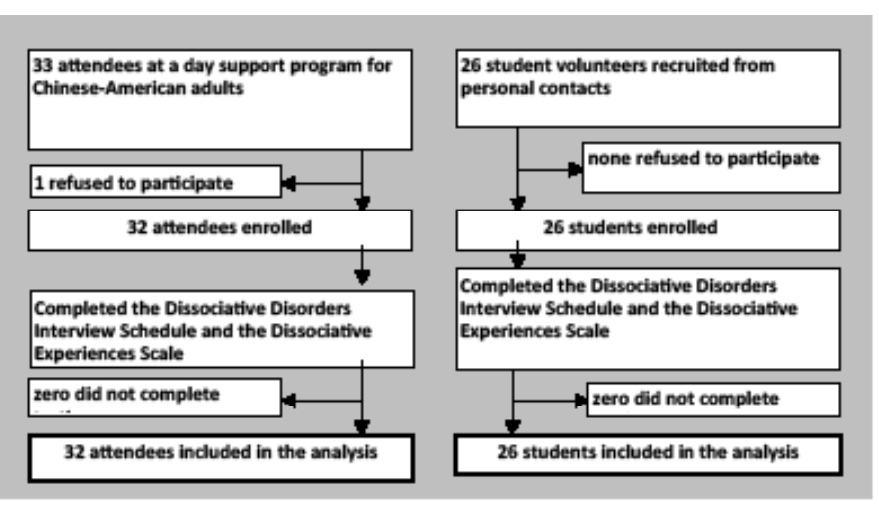

Figure 1. Flowchart of the study.

dissociative disorders. However, dissociative disorder not otherwise specified (DDNOS) was present in $5.2 \%$ of the US participants compared to none of the Shanghai factory workers. This finding is difficult to interpret given the lack of differences on the DES or secondary features of DID. However, overall, it appears that, by most measures of dissociation, the two groups did not differ.

Our findings concerning childhood physical and sexual abuse disconfirmed our hypothesis that there would be no differences between the two groups. Physical and/or sexual abuse was reported by $5.2 \%$ of the US participants and only $0.2 \%$ of the Shanghai factory workers. This could be due to false negatives among the Shanghai participants, or it could be a true difference in trauma rates. However, both groups reported substantially lower rates of physical and sexual abuse than younger survey participants in China and Taiwan in the literature. Thus, although our two groups differed from each other statistically, due to the large sample size in Shanghai, they resembled each other in having much lower rates than younger respondents in the literature.

The other finding of note was the fact that major depressive episode was reported by $12.0 \%$ of the US participants compared to $2.6 \%$ of the Shanghai participants. Although one must again consider reticence to 
report depression in China, there is no compelling reason to expect this, therefore we think that the difference is more likely to be an accurate finding. The causes of this difference cannot be understood using our study design, but they could include sociocultural factors associated with the move from Asia to North America.

The study suffers from a number of limitations. The main one is the small size of the US sample and the fact that it was drawn from one setting in one US city. The two groups were not matched demographically. Additionally, the respondents could have had difficulty understanding the questions, and, as mentioned, childhood trauma might have been under-reported. The strengths of the study are that the same reliable measures were used for both samples, and all the interviews conducted in Chinese were by clinicians fluent in Chinese.

In our view, despite the study's limitations, the findings raise several important questions for future research: have the rates of childhood trauma increased in China over the past three or four decades; and, inversely, are the rates of childhood trauma truly lower among older adults, or are the older adults simply more reticent to report? The higher rates of reported trauma in our US participants could be due to either increased trauma, or reduced reluctance to report due to the influence of American culture. Additional studies with large sample sizes should be conducted to better understand these possibilities.

\section{References}

1. Fan Q, Yu J, Ross CA, Keyes BB, Dai Y, et al. (2001) Teaching Chinese psychiatrists to make reliable dissociative disorder diagnoses. Transcult Psychiatry 48: 473-83. [Crossref]

2. Ross CA, Keyes BB, Xiao Z, Yan HQ, Wang Z, et al. (2005) Childhood physical and sexual abuse in China. $J$ Child Sex Abus 14: 115-126. [Crossref]

3. Ross CA, Keyes BB, Xiao Z, Yan HQ, Wang Z., et al. (2008) A cross-cultural test of the trauma model of dissociation. J Trauma Dissociation 9: 35-50. [Crossref]

4. Xiao Z, Yan H, Wang Z, Zou Z, Xu Y, et al. (2006) Trauma and dissociation in China. Am J Psychiatry. 163: 1388-1391. [Crossref]

5. Xiao Z, Yan H, Wang Z, Zhou Z, Xu Y, et al. (2006) Dissociative experiences in China. J Trauma Dissociation 7: 23-38. [Crossref]

6. Zhang X, Yu J, Zhang Y, Ross CA, Keyes BB, et al. (2011) The reliability and validity of the Chinese version of the Dissociative Experiences Scale. Chinese J Clin Psychiatry. 21: 308-311.

7. Chen J, Dunne HP, Han P (2004) Child sexual abuse in China: A study of adolescents in four provinces. Child Abus Negl 11: 1171-1186. [Crossref]

8. Li X, Wang Z, Hou Y, Wang Y, Liu J, et al. (2013) Effects of childhood trauma on personality in a sample of Chinese adolescents. Child Abuse Negl 38: 788-796. [Crossref]

9. Lin D, Li X, Fan X, Fang X (2011) Child sexual abuse and its relationship with health risk behaviors among rural children and adolescents in Hunan, China. Child Abuse Negl 35: 680-687. [Crossref]

10. Shen L, Zhang Y, Liang W, Zhang Y (2013) Investigation of child maltreatment: Survey among junior school pupils in Henan province of China. Asia-Pac Psychiatry 7: 85-90. [Crossref]

11. Yen C, Yang MS, Yang MJ, Su YC, Wang MH, et al. (2008) Childhood physical and sexual abuse: prevalence and correlates among adolescents living in rural Taiwan. Child Abus Negl 32: 429-438. [Crossref]

12. Bernstein EM, Putnam FW (1986) Development, reliability, and validity of a dissociation scale. J Nerv Ment Dis 174: 727-735. [Crossref]

13. Carlson EB, Putnam FW, Ross CA, Torem M, Coons P, et al. (1993) Predictive validity of the Dissociative Experiences Scale. Am J Psychiatry 150: 1030-1036.

14. Van IJzendoorn MH, Schuengel C. (1996) The measurement of dissociation in normal and clinical populations: Meta-analytic review of the Dissociative Experiences Scale. Clin Psychol Rev 16: 365-382.

15. Ross CA, Duffy CMM, Ellason JW (2002) Prevalence, reliability, and validity of dissociative disorders in an inpatient setting. J Trauma Dissociation 3: 7-17.

16. Ross CA, Miller SD, Reagor P, Bjornson L, Fraser GA, et al. (1990) Structured interview data on 102 cases of multiple personality disorder from four centers. $\mathrm{Am} J$ Psychiatry 147: 596-601. [Crossref]

17. Ross CA, Ness L (2010) Symptom patterns in dissociative identity disorder patients and the general population. J Trauma and Dissociation 14: 224-235. [Crossref]

18. Ross CA, Schroeder E, Ness L. (2013) Dissociation and symptoms of culture-bound syndromes in North America: A preliminary study. J Trauma Dissociation 14: 224-235. [Crossref]

19. Yu J, Ross CA, Keyes BB, Li Y, Dai Y, et al. (2010) Dissociative disorders among Chinese inpatients diagnosed with schizophrenia. J Trauma Dissociation 11: 358-372. [Crossref]

20. American Psychiatric Association (1994) Diagnostic and statistical manual of mental disorders, (4th edn.) Washington: Am Psychiatric Assoc.

Copyright: (C2016 Ross CA. This is an open-access article distributed under the terms of the Creative Commons Attribution License, which permits unrestricted use, distribution, and reproduction in any medium, provided the original author and source are credited. 